\title{
ELECTROSTATIC REPULSION BETWEEN HIGHLY INJECTING METALS: AN EXPERIMENTAL INVESTIGATION
}

\author{
C. T. DERVOS, Ass. Professor \\ Division of Electroscience, Department of Electrical and Computer Engineering, \\ National Technical University of Athens, 9 Iroon Polytechniou str, 15773 \\ Zografou, Athens, Greece
}

(Received 10 November 1994; Revised 14 May 1996; In final form 17 June 1996)

This paper is an experimental investigation of the major implications brought in the crossing resistance of mechanically contacted metals when operating in the high electronic injection regime, i.e., steady state interfacial voltage values greater than the max. acceptable level determined by specifications and less than the melting voltage. Under such operating conditions, monolayers of positive ions may be formed within interfacial cavities filled by the material from the surrounding space. The dominating ion neutralization process on the cathode controls the formation of "Helmholtz" inner layers at the metal cathode+oxide +gas interface. The presence of a positive ion monolayer over the cathode electrode will tend to reduce the field threshold required for electronic field emission and affect the overall currentvoltage characteristics.

The response of contacts operating under high charge injection is monitored on an injected charge vs. interfacial field phase space, which clearly demonstrates non-linear conductivity phenomena and hysteresis effects for the examined structures. Experimental results indicate convincingly the importance of the dielectric media around highly injecting metal contacts. The excessive positive ion formation may also account for the experimentally observed electrostatic repulsion between highly injecting metal contacts. Under low contact pressure situations spontaneous separation of the current carying electrodes may occur. During a spontaneous contact breaking process, the transient current and voltage profiles across the metal contacts have been monitored using state-of-the-art data logging systems. The importance of the oxide capacitance and (time varying) gap capacitance on $V(t)$ and $I(t)$ profiles has been discussed. The obtained results provide evidence for the positive ion layer formation over the cathode electrodes. 


\section{INTRODUCTION}

\subsection{Implications of Positive Ion Layer Formation Across Highly Injecting Metal Contacts}

The injection of electrons from a metal electrode at the interface of the surrounding gas is a phenomenon of primary importance concerning the initiation and evolution of dielectric breakdown [1]. This phenomenon is practically met during the opening stage of all kinds of power switches, which may be operated in air, vacuum, liquid insulating media, i.e., mineral oil, and insulating gasses, i.e., $\mathrm{SF}_{6}$ environment. Direct transfer of electrons from the Fermi level of the metal (cathode) towards the surrounding material is theoretically enabled at very high electric fields as predicted by Fowler and Nordheim for field electron emission from metals into vacuum [2]. For typical metal electrodes, the effective work function, $\phi$, turns out to be of the order of $4 \mathrm{eV}$ implying enormous field application for injection to occur (typical value $45 \mathrm{MV} / \mathrm{cm}$ ). Although such high electric fields are rarely met in practice, the phenomenon of electronic injection is frequently observed. This may be related to the growth of a thin semi-insulating film on the cathode, consisting of a monolayer of positive ions, either ionized impurities or those of the pure gas. The aforesaid dipole layer formation significantly reduces the potential barrier between the metal and the surrounding gas allowing Schottky emission of electrons from the metal into the conduction band of the surrounding media, as shown in Figure 1. It has been estimated that a positive ion density of about $10^{14} \mathrm{~cm}^{-2}$ will be adequate for the initiation of the above effects on a typical metal surface covered by $1 \mathrm{~nm}$ thick oxide overlayer having a relative dielectric constant $\varepsilon_{\mathrm{r}}=10$, [3].

It is worth noticing that if a layer of positive ions is to be formed on the oxide-gas or metal-gas interface, the accumulation period for the ions will have to be significantly smaller than the lifetime period of the ion layer on the metallic surface. The accumulation period depends on the available positive ion concentration in the gas; this is strongly increased whenever ionization mechanisms are involved, i.e., presence of ionizing radiation or application of high electric fields. On the other hand, the life-time of the ions is determined by the dominating ion neutralization process at the metal-gas or oxide-gas interface. After the neutralization and removal of an ion, its site may be accommodated by one of the nearby accumulated 


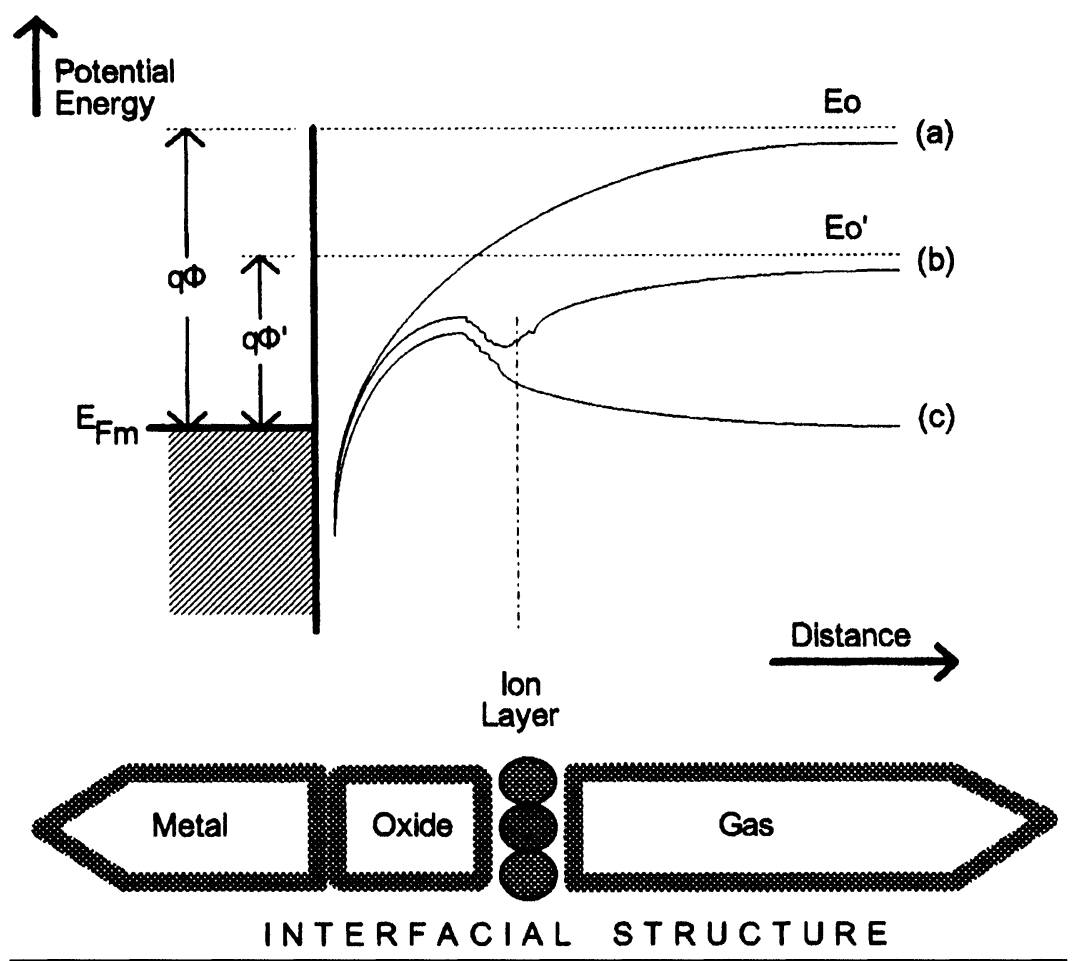

FIGURE 1 Dependence of the effective work function, $\phi$, on the metal surface condition. (a) Ideal metal-vacuum interface. (Eo is the vacuum level).

(b) Metal-oxide-ion layer-vacuum structure. (Eo' is the corresponding vacuum level).

(c) Same structure as in case (b) having externally applied potential. (The metal electrode is positive). The constituent layers of the interfacial structure are indicated underneath. The arrows in the gas and metal layers imply that these materials extend significantly towards these directions.

ions, thus restarting the neutralization cycle. The process is self-repeated provided there is a negative potential on the metal electrode and an available positive ion concentration in the surrounding space at the vicinity of the metal electrode. Some of the possible ion neutralization processes for a metal-gas or a metal-liquid dielectric interface could be the following: Resonance tunnelling, Auger neutralization, and resonance coupling [4]. Assuming that positive ion layers do form on metal cathodes and that all of the above ion neutralization processes are applicable at the given metal- 
ion system, it is more likely that only one transfer mechanism will be the dominant one, although this need not be always the case. Note that slower processes for electron transfer to the ions will be associated with significantly longer lifetime period, thus allowing more time span for the ion accumulation to take effect and regenerate the dipole layer immediately after neutralization.

This work is focused on the implications arising for the positive ion layer formation on the cathode electrode and provides relevant experimental evidence indicating its existence.

\subsection{The Asperity Contact Model and the Possible Nucleation Sites for the Ion Layer}

Although a number of possible ion neutralization processes can theoretically be pointed out for oxidized metallic surfaces, we can not be certain whether such ionic layers do occur in practical applications. If a layer of positive ions is to be formed on the interface, it will produce an electric dipole layer across the metallic oxide overlayer. Polarization effects on a large scale are hardly noticed, and as such, they are not considered systematically in practical power applications. The undertaken experimental work is based on the investigation of mechanically contacting metal surfaces sustaining high charge injection rates, (i.e., high ampacity at moderate contact areas) and aims to demonstrate some of the implications introduced by the positive ion layer formation over a cathode electrode.

The examined systems are energized by a standard $50 \mathrm{~Hz}$ sinusoidal power source and the practical aspect of the investigated structure covers all types of stationary and switching contacts in power networks. For such contacts, it is commonly accepted that the electronic charges are injected via specific spots across the interface [5], [6]. The overall area of these injection centers determines the effective contact area. The distribution of the effective contact spots, as well as the effective contact area, depends upon surface geometry, degree of polish, hardness of materials, mechanical pressure, and phase transitions with increasing temperature. The effective contact area is usually smaller than the apparent contact area [6], [7]. However, when an arc forms around the interface this argument may not be valid since field emitted electrons in the surrounding gas increases significantly the effective contact area [7]. 
It would be interesting to specify the possible contact regions where the aforesaid ion layer forms. As indicated in Figure 2, these ions are possibly generated by an impact ionization process in cavities formed between the electrodes and move along the field lines towards the cathode. It is expected that these cavities will be filled with material from the surrounding space. Therefore, the operating environment (i.e., pressurized $\mathrm{SF}_{6}$, air at atmospheric pressure, vacuum) is very important as it predetermines the mean electron free path and consequently the ionization probability. Considering that in practical applications the gap across non-ideally contacting regions in the apparent contact area is significantly smaller than $1 \mathrm{~mm}$, one can argue that the ion generation rate will be drastically reduced at lower pressures, because the mean free path for electrons becomes several $\mathrm{cm}$,

A NODE

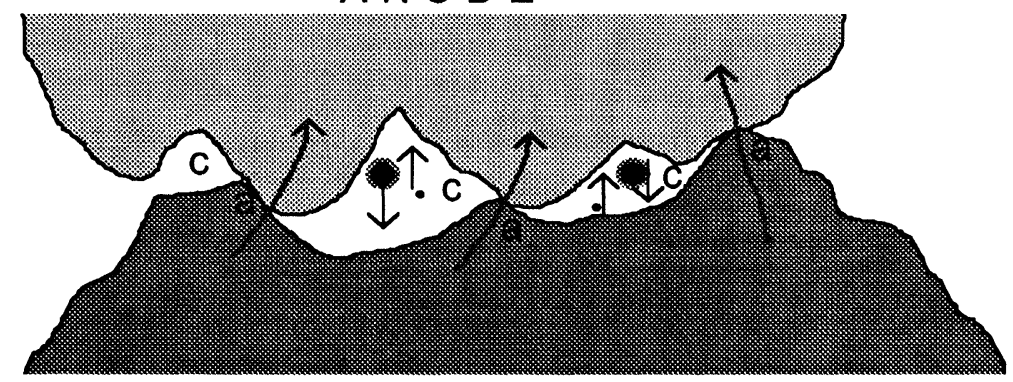

C ATHODE

positive ion

- free electron

\section{a ideally contacting spots}

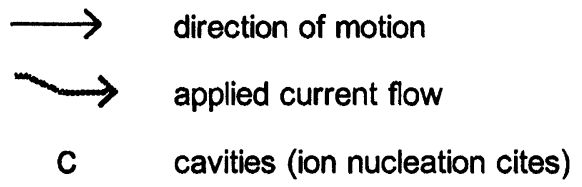

C cavities (ion nucleation cites)

FIGURE 2 Due to material surface roughness cavities are formed at the interface of mechanically contacting metals. These are filles with the material of the surrounding space and are expected to play a significant role during high electronic injection intervals since they may transform in effective positive ion nucleation sites. The ideally contacting spots -a spotswill account for the ohmic behaviour of the contact while cavity formation induces capacitive effects. 
[8] and there is not sufficient space for the ions to be generated in the interfacial cavities. On the other hand, the ion layer formation over the highest field regions of the cathode will increase the overall electronic emission efficiency. Therefore, from all ion layer nucleation sites, only those within the apparent contact area will mainly control the Schottky emission. It should also be pointed out that for the closed switch contacts, the ion layer cannot form within the ideally contacting "a" spots of Figure 2 (i.e., the majority of the effective contact area) as there is no available space for the ions to be placed there. This area will play a significant role during the transient state of contact opening, providing unexposed metal spots for rapid ion layer build-up, thus being an ideal example for the experimental investigation of the above effects.

\section{EXPERIMENTAL}

\subsection{Measurements Across Stationary Metal Contacts Indicating Current Instabilities Due to Ionization Effects in the Surrounding Space}

The examined structures consisted of Oxygen Free High purity Copper (OFHC) contacts having a hemispherical-plane electrode assembly. Their clamping force was maintained during measurements by employing axial springs displaced by micrometric potentiometers giving a constant clamping force of up to 1 Newton. To ensure high electronic injection at the interface, apparent contact areas of the order of $0.5 \mathrm{~mm}^{2}$ (optically measured) were subjected to stable sine wave currents of 160 A RMS or more. According to specifications IEC 0660, high injection across mechanically contacted metals is practically met when the potential drop across the interface becomes greater than $50 \mathrm{mV}$. The "melting voltage" is the potential difference at the interface capable of establishing "a molten bridge" between the solid surfaces [9]. This voltage value depends on the material. It is greater than $50 \mathrm{mV}$ by at least one order of magnitude and introduces the upper potential difference limit for a solid metal contact to operate under high charge injection without phase transitions to occur.

A two channel data logger was specifically realized for the purpose of such experiments and was interfaced with the data-bus of an Intel-386 
microprocessor system. Digital signal tracking enabled real-time measurements with sampling rates as fast as 40 MSPS having maximum reading error $0.1 \%$. In-situ amplification by $40 \mathrm{~dB}$ enabled weak signal monitoring. The response of the employed systems was monitored by the simultaneous recordings of the

- stimulating applied sinusoidal potential and

- net charge injected across the contacting joints.

The ionization occurrence within cavities located between the mechanically contacted interfaces initiates a chaotic behavior [10] which can be visualized by the implementation of the appropriate observation diagram, (i.e., phase space) [11] having the input signal for the examined component across the horizontal axis (interfacial field $\propto$ voltage) and its response on the vertical (injected charge $\propto$ current). A detailed approach concerning the applicability of such methods in monitoring current instabilities for industrial components is given in [12].

To demonstrate current instabilities introduced in the overall current profile by some additional charge transport mechanisms, i.e., field emission among non-ideally contacting spots in the apparent contact area, the following experiments were performed: The current-voltage characteristics of highly injecting hemispherical copper electrodes, which had suffered ageing after several years of operation in a power network were monitored over an operation interval of 25 periods (ac $50 \mathrm{~Hz}$ ) under two different operating conditions:

- atmospheric environment and

- high vacuum, $10^{-6}$ mbar.

The high-vacuum environment was produced by a 450 liters/sec, water cooled, oil diffusion pump operated with Apiezone Dow Corning 704 fluid, backed by a two stage rotary pump. The vacuum chamber consisted of stainless steel 1.4306 [AISI $304 \mathrm{~L}$ ] and 1.4429 [AISI $316 \mathrm{LN}$ ] defined in DIN 17440 and glass S801.51. Viton and OFHC were used as sealing materials. Cooled high-current electrical feed throughs were used for the application of high currents in structures under test. The total pressure in the chamber was monitored using a Bayard-Alpert ionization gauge [8]. This was found to be $10^{-6}$ mbar during measurements. 
It was established experimentally that identical stimulating currents and mechanical contact pressures for the two working environments produced vastly different results during the high injection intervals, i.e., maximum applied electric field of either polarity. Figures $3 \mathrm{a}$ and $3 \mathrm{~b}$ show the obtained results. Same scales have been applied in both graphs for comparison purposes. It can be seen that the response scattering has a greater degree of uncertainty when the system is operating under atmospheric conditions. This could be attributed to the non-linear conductivity processes, i.e., field emitted electrons due to the increased injection efficiency whenever an ionic layer forms. This is to be expected as the probability for the ion generation differs between air and vacuum. High vacuum increases the mean electron free path and consequently reduces the concentration of ions generated in the gap by an impact ionization avalanche process [13]. In a high vacuum environment, the reduction of the available ion concentration between non-ideally contacted spots will tend to suppress the formation of the ionic layer since the neutralization occurs at a constant rate but there does not exist sufficient ion concentration to rebuild the surface ion layer immediately. It is also worth noticing that the above phenomenon is reversible, i.e., the introduction of air in a vacuum chamber reforms the observed current-voltage trajectories on the phase space and response scattering becomes evident again.

The observed current instabilities for contacts operating in atmosphere cannot be attributed to phase transitions since these occur at much greater time scales and higher interfacial voltage values. In addition, due to the limited heat dissipation in a vacuum, one would expect that the current instabilities related with melting processes would dominate when operating in vacuum-which does not occur.

\subsection{Measurements Across Highly Injecting Stationary Metal Contacts Indicating Electrostatic Repulsion Between the Contacting Joints}

Another implication possibly rising by the ion layer formation is the electrostatic repulsion, which has been reported to occur at least for the case of arcing metal-metal contacts [14]. Practical applications treat such problems either by increasing the applied force between the contacting joints, 
a

Q

arbitrary

units

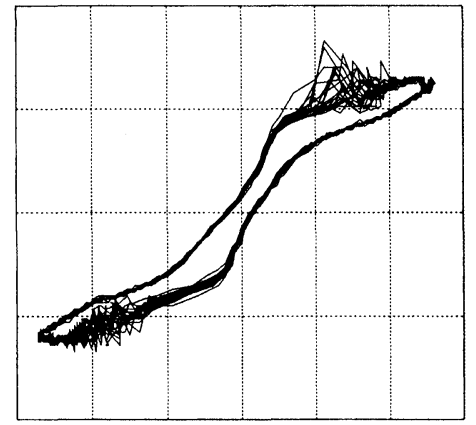

E arbitrary units

b

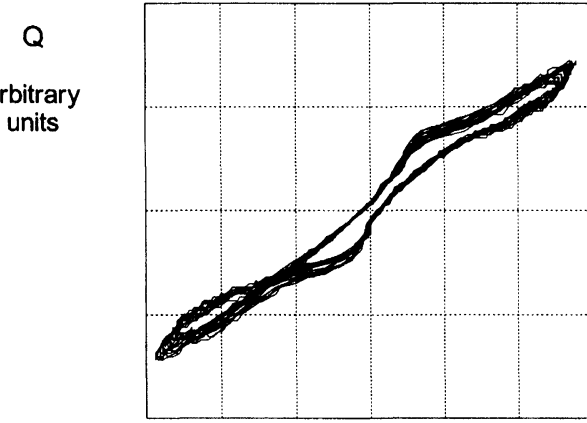

E arbitrary units

FIGURE 3 Successively recorded 25 loops of injected charge, Q, vs. interfacial field, E, across highly oxidised copper-brass contacts sustaining $200 \mathrm{~A} \mathrm{rms}-50 \mathrm{~Hz}$ currents.

(a) Laboratory environment.

(b) High vacuum $\left(10^{-6}\right.$ mbar).

Same operating conditions and scaling factors apply throughout for comparison purposes. The resulting "rounded" loop could be brought-up as a result of a nonlinear conductivity structure (with considerable interfacial capacitance value) subjected to an oscillating field. Note area reduction and overall loop recovery when operating in vacuum. 
or by employing specific designs, such as the "knife type" of no load switches.

The retarding forces developing across highly injecting stationary metal contacts might be introduced by either of the two complementary processes described below:

$\boldsymbol{a}$. Excessive positive ion accumulation on the surface of the cathode will result in an electrostatic force application since the mobile ions are continuously repelled from the anode and are screened away from the cathode by the presence of the surface ion layer. The retarding force depends on the average impinging rate of positive ions against the cathode, the mass number of these ions, their charge (singly or doubly ionized), and their momentum. This may become particularly important for components operating with dielectric gases where some of the produced fragments may be characterized by relatively large ion mass numbers (e.g., typical fragments for $\mathrm{SF}_{6}$ when ionized by $100 \mathrm{eV}$ electrons are: $\mathrm{SF}_{5}^{+}, \mathrm{SF}_{4}{ }^{+}, \mathrm{SF}_{3}{ }^{+}$, $\mathrm{SF}_{2}{ }^{+}, \mathrm{SF}^{+}, \mathrm{S}^{+}, \mathrm{F}^{+}, \mathrm{SF}_{4}{ }^{++}, \mathrm{SF}_{3}{ }^{++}, \mathrm{SF}_{2}{ }^{++}$, etc; molecular ions are not observed) [15].

$\boldsymbol{b}$. Electrons having high kinetic energies impinge on the anode surface introducing repulsive forces while loosing their excess energy either by thermalization or metal erosion processes (e.g., sputtering).

No matter which of the above processes introduces the dominating retarding component among the contacting joints, it is a fact that highly injecting mechanically contacted metals are electrostatically repelled [14]. This phenomenon occasionally becomes noticeable in the external circuit provided the metallic joints are weakly attached. In that case, spontaneous contact opening during operation may be instantly observed during the high injection intervals (i.e., maximum current values of either polarity). Figure 4 convincingly demonstrates the effect for the case of a stationary metal contact operating under ac currents and low contact pressure situations. The observed current-voltage instabilities may be explained as following: During the intervals of large current densities-and therefore, excessive positive-ion impinging rate against the cathode-the retarding forces may instantly exceed the clamping force between the joints and therefore, a spontaneous separation process may be initiated. As the joints are brought further apart, the field in the gap is drastically reduced, thus minimizing the net ionization rate. This results in reducing the available positive ion concentration at the vicinity of the cathode, increasing the accumulation period and therefore leading to the elimination of the ion 


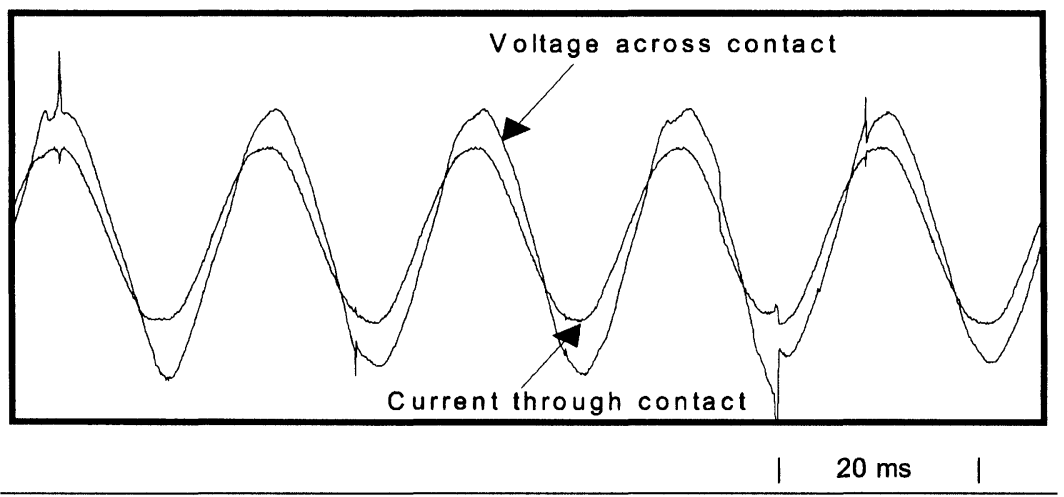

FIGURE 4 Current and voltage profiles traced across highly injecting hemispherical copper contacts. Note instability occurrence implying instant increase of contact resistance (increased voltage and reduced current). This could be related to spontaneous separation process across mating joints. Clamping force $=0.2 \mathrm{~N}$. Apparent contact area $=0.5 \mathrm{~mm}^{2}$. Current through contact $=230 \mathrm{~A} \mathrm{rms}, 50 \mathrm{~Hz}$. Steady state peak voltage value across the junction $=$ 0.7 Volts.

monolayer formation. In that case, the retarding force no longer exists and the clamping force takes over to bring the joins back into contact again. In power switch applications, this process may repeat itself occasionally, producing the effect of "a flickering contact" with current instabilities. This may also account for the "rattling noise" produced by the joins of the contacts, which have suffered mechanical ageing in their clamping mechanisms.

It was also experimentally confirmed that the probability for observing the aforesaid phenomenon also depends on other parameters such as environmental conditions, i.e., humidity, and overall contact temperature. Such effects are usually intensified during the initial stages of operation, when the temperature of the adjacent metals is much lower compared to their steady-state temperature value [16]. The above experimental observations are within the framework of the proposed model since humidity will affect the ionization rate, while temperature enhancements may interfere in a much more complicated manner, i.e., affect injection efficiency, alter humidity in cavities, vary the electron mean free path and control the ionization rate, increase oxidation kinetics, and alter the neutralisation cycle, etc. 


\subsection{Transient Signals Recorded During Contact Opening Which Indicate the Existence of Positive Ion Layers}

In order to demonstrate the existence of the surface ion layer on the cathode electrode, the following experiment was also performed: Two contact joints were placed on a shaft and could be displaced along their axial direction whenever repulsive forces were applied between them. Their clamping force was maintained at a constant low value (of the order of 0.1 Newton) while the current through the contacting joints was increased stepwise until spontaneous separation occurred. The transition from state "CLOSE" to state "OPEN" was always accompanied by strong arcing phenomena indicating plasma formation between metal electrodes. During the passage from state "CLOSE" to "OPEN", the corresponding potential across the contacting joints and the net charge transport, i.e., overall current profile, were synchronously digitally recorded and the results obtained are given in Figure 5. In the presented example, it takes about two periods of the stimulating signal $(50 \mathrm{~Hz})$ to accomplish this transition. Note that during the transient period two distinct operating conditions may be noticed:

- Prior contact opening, finite intervals exist where the potential difference across the contacts is significantly increased, at absolute value. Synchronously current peaks-significantly greater than the steady-state peak current values on the opposite direction are observed.

- The potential profile across the joints has reached the open circuit value, Voc, which is determined by the specifications of the employed power source, while the corresponding current value is zero. During these intervals, the electrical contact is fully opened.

The response observed during permanent contact opening is further discussed in the following paragraphs.

\subsubsection{Potential Profile}

During the initial separation stages, the effective contact area is reduced and fresh unexposed areas of the cathode appear in the plasma gap. These (oxidized) surface areas will be instantly covered by the positive ion layer in the process to neutralization. The presence of the ions on the oxide overlayer will induce mirror electron charges at the metal-oxide interface 
a

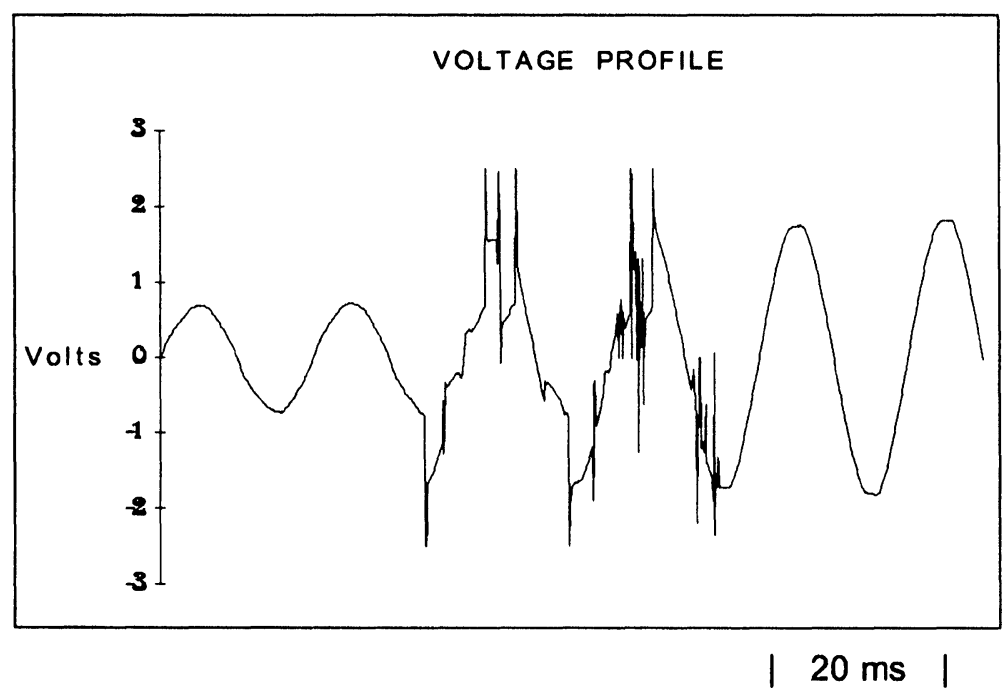

b

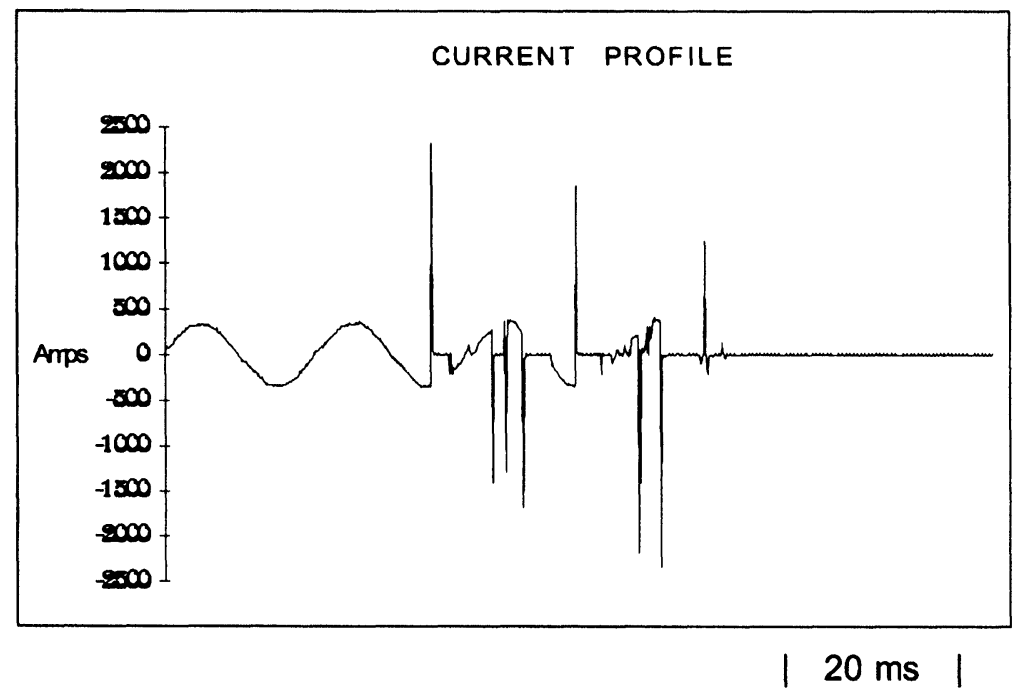

FIGURE 5 Voltage and corresponding current profiles during the spontaneous electrostatic separation of highly injecting contacts. A synchronous digital recording 50 ksps has been applied. 
of the cathode. This dipole layer structure is schematically given in Figure 6 and is frequently referred in literature as the "Helmholtz" ion monolayer, [17]. To evaluate the voltage difference, $\Delta \mathrm{V}$, superimposed by the positive ion layer on the overall potential across the joints, the following quantities will have to be defined: The oxide capacitance, $\mathrm{C}_{\mathrm{ox}}$, refers to all cathode spots within the apparent contact area covered by the fresh ion layer (note that the ion layer initially forms over the highest field spots). Oxide capacitance will be given by an equation of the following form:,

$$
\mathrm{C}_{\mathrm{ox}}=\varepsilon_{o} \cdot \varepsilon_{r} \cdot \frac{\mathrm{S}}{\lambda}
$$

where, $\varepsilon_{\mathrm{o}}$ is the permittivity of free space, $\varepsilon_{\mathrm{r}}$ is the relative permittivity for the thin metal oxide overlayer (possibly of the order of 10 since thin oxide layers have semiconducting properties), $\mathrm{S}$ is the effective contact area where fresh ion layers may be formed (smaller than the optically measured apparent area values $\approx 0.5 \mathrm{~mm}^{2}$ ), and $\lambda$ is the average width for the oxide

$(-)$

VAPP

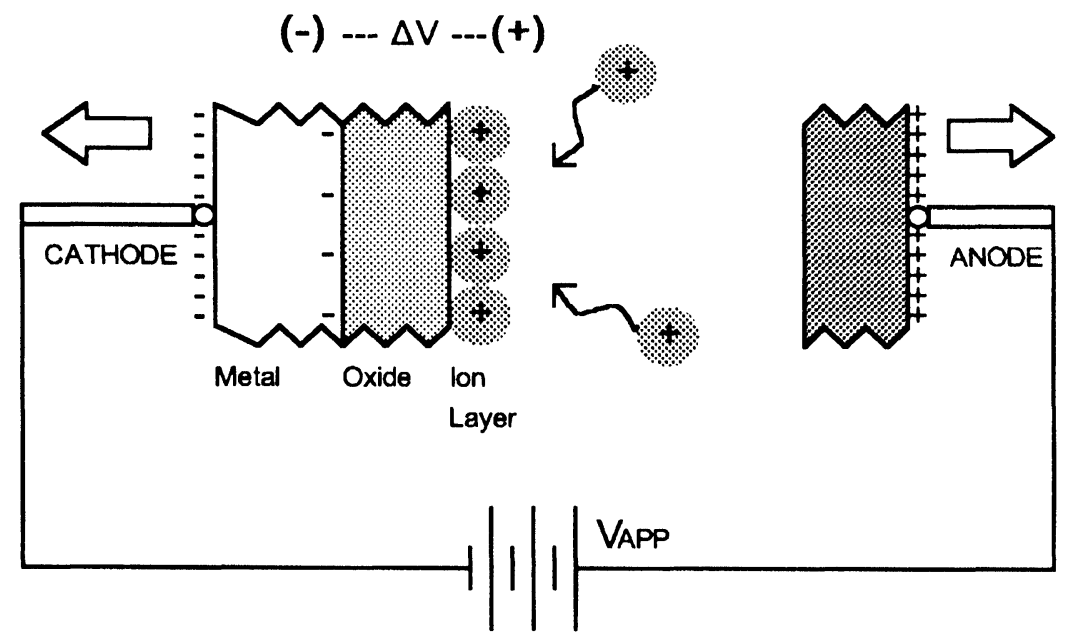

FIGURE 6 Charge distribution across a contact microstructure during the initial stages of spontaneous separation. The net potential difference across the interface is given by $\mathrm{V}_{\text {TOT }}=$ $\mathrm{V}_{\mathrm{APP}}+\Delta \mathrm{V}$, where $\Delta \mathrm{V}$, is determined by the positive ion layer and $\mathrm{V}_{\mathrm{APP}}$, by the employed power source. The arrows indicate possible directions of motion. 
overlayer (typical value is of the order of 40 Angstroms since charge transfer occurs by quantum mechanical tunnelling even at moderate field application).

The voltage increase, $\Delta \mathrm{V}$, due to the presence of the ion charges is given by the equation,

$$
\Delta \mathrm{V}=\Delta \mathrm{Q}_{(\mathrm{IONS})} / \mathrm{C}_{\mathrm{ox}}
$$

where $\Delta \mathrm{Q}_{\text {(IONS) }}$ is the total charge from the ions in the newly developed layer and is given by,

$$
\Delta \mathrm{Q}_{(\mathrm{IONS})}=\mathrm{N}_{(\mathrm{IONS})} \cdot \kappa \cdot \mathrm{q} \cdot \mathrm{S}
$$

where $\mathrm{N}_{\text {(IONS) }}$ is the surface concentration for the ions, $\kappa$ is a coefficient accounting for the singly or doubly ionised fragments, and $\mathrm{q}$ is the electronic charge.

Combining equations (1), (2) and (3), one can obtain,

$$
\Delta \mathrm{V}=\frac{\mathrm{N}_{(\mathrm{IONS})} \cdot \kappa \cdot \mathrm{q} \cdot \lambda}{\varepsilon_{o} \cdot \varepsilon_{r}}
$$

Substituting for the accumulating surface ion concentration the typical value quoted in literature, $\mathrm{N}_{(\text {IONS) }}=10^{14} / \mathrm{cm}^{2}$ [3], assuming singly charged ions $(\kappa=1), \varepsilon_{\mathrm{r}}=5$ and $\lambda=40$ Angstroms, one can get that the voltage increase $\Delta \mathrm{V}$ is of the order of 1 Volt (independent of the exposed area). This is in remarkable agreement with the typical results shown in Figure 5a, where the voltage build-up during the overcurrent production is found to exceed the open circuit value Voc by approximately $1 \mathrm{~V}$.

According to equation 4 and the above analysis, greater oxide width results in higher voltage peaks. However, there is an upper limit that is introduced by the requirements for a good ohmic behavior of the structure. If we consider thin oxide structures that enable charge transfer by quantum mechanical tunnelling, the maximum oxide width will have to be of the order of 100 Angstroms [18]. However, in practical applications the interfacial layer width may extend beyond these levels, in which case, there is a required voltage threshold for conduction to occur. Now the charge transport through the interfacial layer is accomplished by field emission 
[19] or field ionization [20] processes. Strongly oxidized surfaces will produce higher voltage build-up during the initiation of a separation process. Assuming singly charged ions and substituting for $\varepsilon_{\mathrm{r}}=5$ and $\lambda=1$ $\mu \mathrm{m}$, we conclude that the expected voltage rise $\Delta \mathrm{V}$ due to the positive ion layer formation at a power contact opening process will be of the order of 3600 Volts! This might explain the transient overvoltages produced in power networks during switch opening process.

\subsubsection{Current Profile}

During the initial separating stages of the metal joints, current peaks of the opposite direction are experimentally observed; typical response is given in Figure 5b. To understand the origin of these current surges, the overall capacitance between the effective contact spots-where the fresh ion layer forms-has to be considered. This consists of two terms connected in series:

- the capacitance of the cathode oxide, $\mathrm{C}_{\mathrm{ox}}$

- the capacitance of the gap, $\mathrm{C}_{\text {gap }}$.

The oxide capacitance remains unaltered since its geometry will not be affected at the initial stage of a separation process when the molten bridge is gradually formed. The charges associated with $\mathrm{C}_{\mathrm{ox}}$ are determined by the surface ion concentration, which is constant, provided there is adequate ion concentration in the gap to substitute for neutralized ions.

The capacitance of the gap is formed between the anode electrode and the surface oxide on the cathode and it is inversely proportional to their separating distance, $s$.

$$
\mathrm{C}_{\text {gap }} \propto \frac{1}{S}
$$

The charges stored across the gap capacitance depend on the instant field between the separating joints, while the developing voltage depends on the capabilities of the employed power source. Therefore,

$$
\mathrm{C}_{\text {gap }}=\frac{\mathrm{Q}_{\text {(FIELD GAP) }}}{\mathrm{V}_{(\text {POWER SOURCE) }}}
$$


During the separating stage, the gradual reduction of the field in the gap is balanced by the charge removal. This physical process is schematically given in Figure 7. The rapid reduction of the interfacial charges in the metal electrodes by $\delta Q_{\text {(FIELD GAP) }}$ induces current surges, $\delta \mathrm{I}(\mathrm{t})$, with opposing conventional direction, compared to the current component, $\mathrm{I}_{\mathrm{APP}}$, produced by the external power source during stable "CLOSED" contact operation. The aforesaid charge diminution is inversely proportional to the gap expansion between the joints.

$$
\delta \mathrm{Q}_{(\mathrm{FIELD} \mathrm{GAP})} \propto \frac{1}{\delta \mathrm{s}}
$$

The current pulse produced within interval $\delta$ t relates to charge changes across this capacitance according to the equation.

$$
\delta \mathrm{I}(\mathrm{t})=\frac{\delta \mathrm{Q}_{(\text {FIELD GAP) }}}{\delta \mathrm{t}}
$$

Combining equations (7) and (8), it can be argued that transient current surges, $\delta \mathbf{I}(\mathrm{t})$, are proportional to

$$
\delta \mathrm{I}(\mathrm{t}) \propto \frac{1}{\delta \mathrm{s} . \delta \mathrm{t}}
$$

Therefore, high current surges are expected to occur whenever the joints are rapidly repelled $(\delta t \rightarrow 0)$ at tiny distances $(\delta s \rightarrow 0)$. In the recorded paradigm of Figure $5 \mathrm{~b}$, current surges $\delta \mathrm{I}(\mathrm{t})$ appear to be of the order of $3000 \mathrm{~A}$ for an interval of the order of $50 \mu \mathrm{s}$, while the peak steady-state sine wave current values passing through the contacts are of the order of $400 \mathrm{~A}$.

Closing, it may be argued that in the steady-state operation of practical components, there is continuously applied a sufficient clamping force to ensure stable electrical characteristics, i.e., avoid spontaneous contact opening due to repulsive forces between charge carriers and the electrodes. However, during the switch opening, as the applied contact force between the joints is gradually reduced and the mating surfaces start separating 

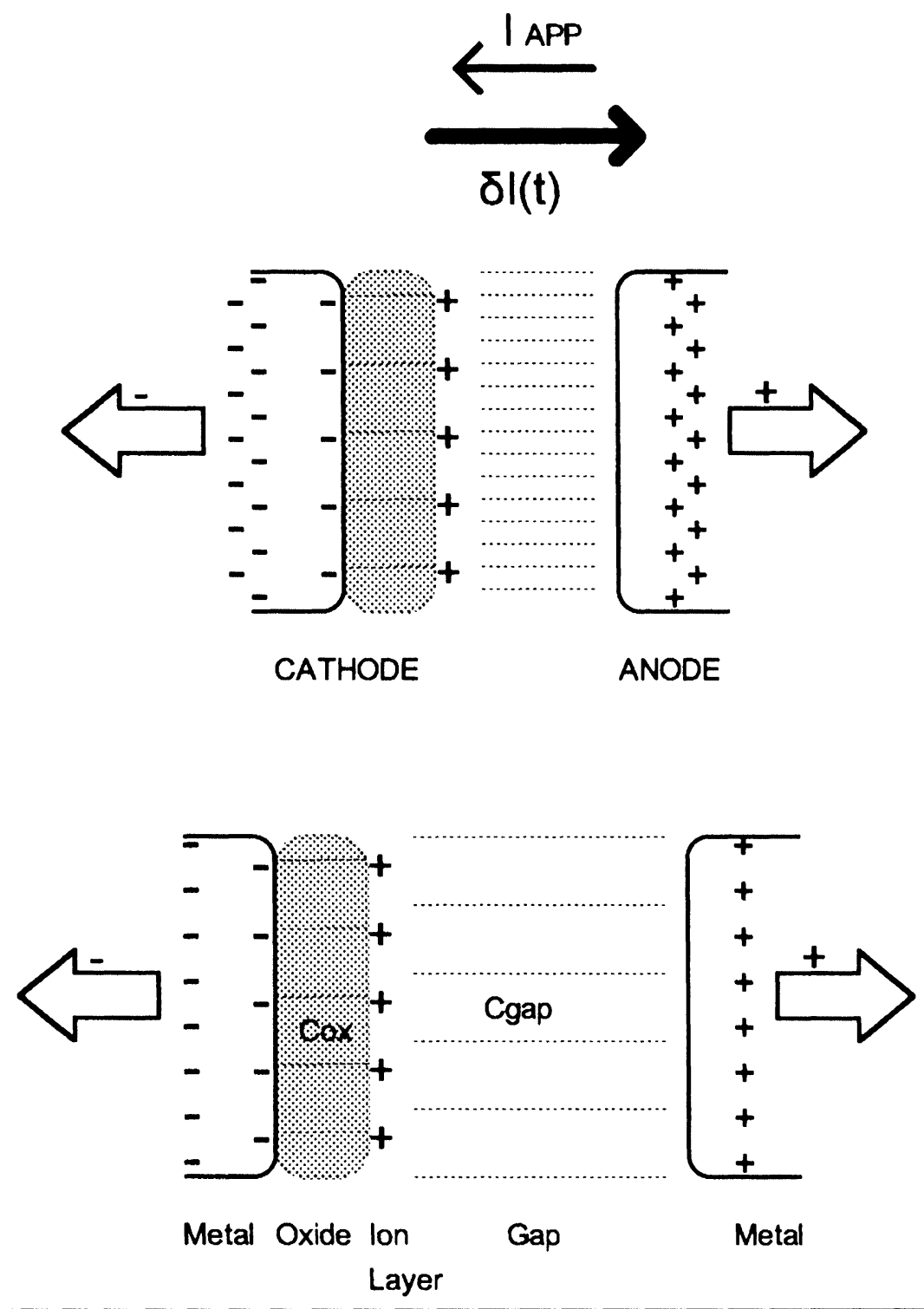

FIGURE 7 Charge distribution during separation process as the gap between the joints is further increased. Arrows along the sides indicate carrier polarity and direction of charge transfer. The intensity of the field lines at the gap is demonstrated by the broken lines. Conventional current flow directions for $\mathrm{I}_{\mathrm{APP}}$ and $\delta \mathrm{I}(\mathrm{t})$ are indicated at the top. 
from each other, the ion layer formation will have to be systematically considered for switching contact reliability and quality control assurance technology.

\section{CONCLUSIONS}

Ion neutralization processes around a metal-gas interface may account for a variety of physical reasons. It is believed that for highly injecting metal contacts, the neutralization processes can give rise to a positive ion monolayer (Helmholtz layer) over the cathode electrodes.

- If such positive ion layers are formed, they will tend to reduce the effective barrier height, altering the performance of current conducting metallic interfaces. The presence of an appropriate gas around mechanically contacted metal surfaces may suppress the ionization rates in interfacial cavities and control barrier lowering conditions whenever required. This might explain the performance of pressurized $\mathrm{SF}_{6}$, frequently adopted in power applications, which is known to minimize the required insulating distances in component fabrication but deteriorates its performance in the presence of moisture, or radiation-induced by-products during power switch operations.

- Self-sustained electrostatic repulsion forces between highly injecting metal contacts may be generated as a result of exceptionally high ion concentration in interfacial cavities by the presence of interfacial ion layers and can be effectively prevented by suppressing the ionization rates. This can be practically attained by increasing the effective contact area. Contact area increase may be practically attained either by employing special geometries between the joints, or by increasing the applied axial pressure. For specified operating conditions, the increase of the contact area reduces the interfacial field values, suppressing the electronic emission rate between the non-ideally contacting spots, and consequently the ionization rate in the gap. The ionization rate in the gap may be further suppressed by controlling the surrounding media, i.e., increase the mean free path of electrons by reducing the atmospheric pressure, or by introducing gaseous dielectrics that are characterized by the highest possible ionization energies. 
- Breaking of an electrical contact produces overcurrents in the external circuit with conventional direction opposite to the applied current direction. In addition, such sharp current pulses have durations of about $50 \mu \mathrm{s}$ and are at least an order of magnitude greater than the steady-state peak current values crossing the interface.

The existence of the aforesaid positive ion layers and related polarization effects will have to be systematically examined in switch contact technology in order to attain control over the transient $\mathrm{I}-\mathrm{V}$ behavior of all kind power contacts in their opening process.

\section{References}

[1] H.R. Zeller, "Breakdown and prebreakdown phenomena in solid dielectrics", IEEE Trans. Elec. Insul., Vol. 22, pp. 115-122 (1987).

[2] Fowler, R. H., Nordheim, L. (1928) Proc. Roy. Soc., (London) A119, p. 173

[3] T.J. Lewis, "Electronic processes in dielectric liquids under incipient breakdown stress", IEEE Trans. Elec. Insul., Vol. 20, pp. 123-132, (1985).

[4] D.K. Roy, "Quantum Mechanical Tunnelling and its Applications", World Scientific, Singapore, (1986)

[5] J.P.B. Williamson, "The microworld of the contact spots", Proc. 27th IEEE Holm Conf. on Electrical Contacts, Vol. 27, pp. 1-10, (1981).

[6] R. Holm, "Electric Contacts, Theory and Applications", 4th Edition, Springer Verlag, Berlin, (1979)

[7] C.T. Dervos, "The reliability of the asperity contact model in determining charge injection across interfaces", Proc. 40th IEEE Holm Conf. on Electrical Contacts, Vol 40, pp. 211-222, Chicago, (1994).

[8] G.F. Weston, "Ultra High Vacuum Practice", Butterworths, London, (1985).

[9] T. Utsumi, "Theoretical and experimental investigations of the dynamic molten bridge", IEEE Trans. Parts, Hybrids, Packag., Vol. 5, pp. 62-68, (1969).

[10] C.A. Pickover, "Pattern formation and chaos in networks", Commun. ACM, USA, Vol. 31, No. 2, pp. 136-151, (1988).

[11] D.C. Arney, B.T. Robinson, "Exhibiting chaos and fragments with a microcomputer", Comput. Math. Appl., Vol. 19, No. 3, pp. 1-11, (1990)

[12] C.T. Dervos and P.T. Fitsilis, "Bifurcation evolution across metal-metal contacts sustaining high charge injection rates", IEEE Trans Comp. Packag. Manuf. Technol., Vol. 17, No 2, pp. 286-294, (1994).

[13] M. Sparks, D.L. Mills, R. Warren, T. Holstein, A.A. Maradudin, L.J. Sham, D.F. King, "Theory of electron-avalanche breakdown in solids", Phys. Rev. B., Vol. 24, pp. 3519-3536, (1981).

[14] J.P. Chabrerie, J. Devautour, A.M. Gouega and Ph. Teste', "A sensitive device for the measurement of the force exerted by the arc on the electrodes", Proc of 40th Holm Conference on Electrical Contacts, Vol. 40, pp. 261-267, Chicago, USA, Oct. 17-19, (1994).

[15] J.A. Koprio, "Detecting sulfur hexafluoride", Balzers Application Report, BG 800008 AE (8610).

[16] C.G. Karagiannopoulos, P.D. Bourkas, C.T. Dervos and C.A. Kagarakis, "Physical interpretations concerning nonlinear conductivity phenomena across no-load switch- 
ing contacts", IEEE Trans Comp., Hybrids, Manuf. Technol., Vol. 14, No. 1, pp. 137-142, (1991).

[17] Morrison, "Electrochemistry at Semiconductor and Oxidised Metal Electrodes", Plenum Press, New York, (1980).

[18] J.C. Simmons, "Generalised formula for the electric tunnel effect between similar electrodes separated by a thin insulating film", J. Appl. Phys., Vol 34, No 6, pp. 1793-1803, (1963).

[19] B. Halpern, R. Gomer, "Field emission in liquids", J. Chem. Phys., Vol. 51, pp. 1031-1047, (1969).

[20] B. Halpern, R. Gomer, "Field ionisation in liquids", J. Chem. Phys., Vol. 51, pp. 1048-1056, (1969). 

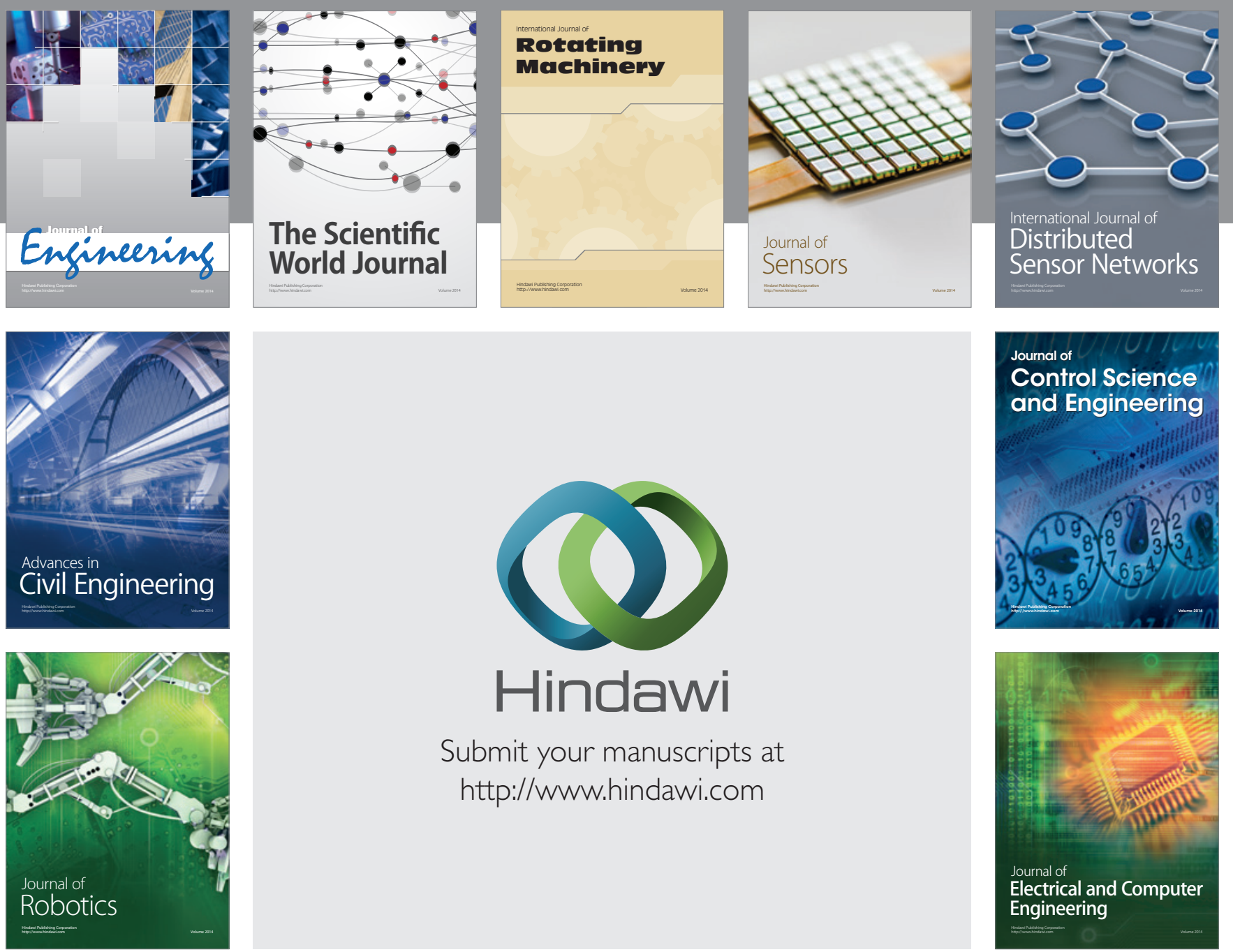

Submit your manuscripts at

http://www.hindawi.com
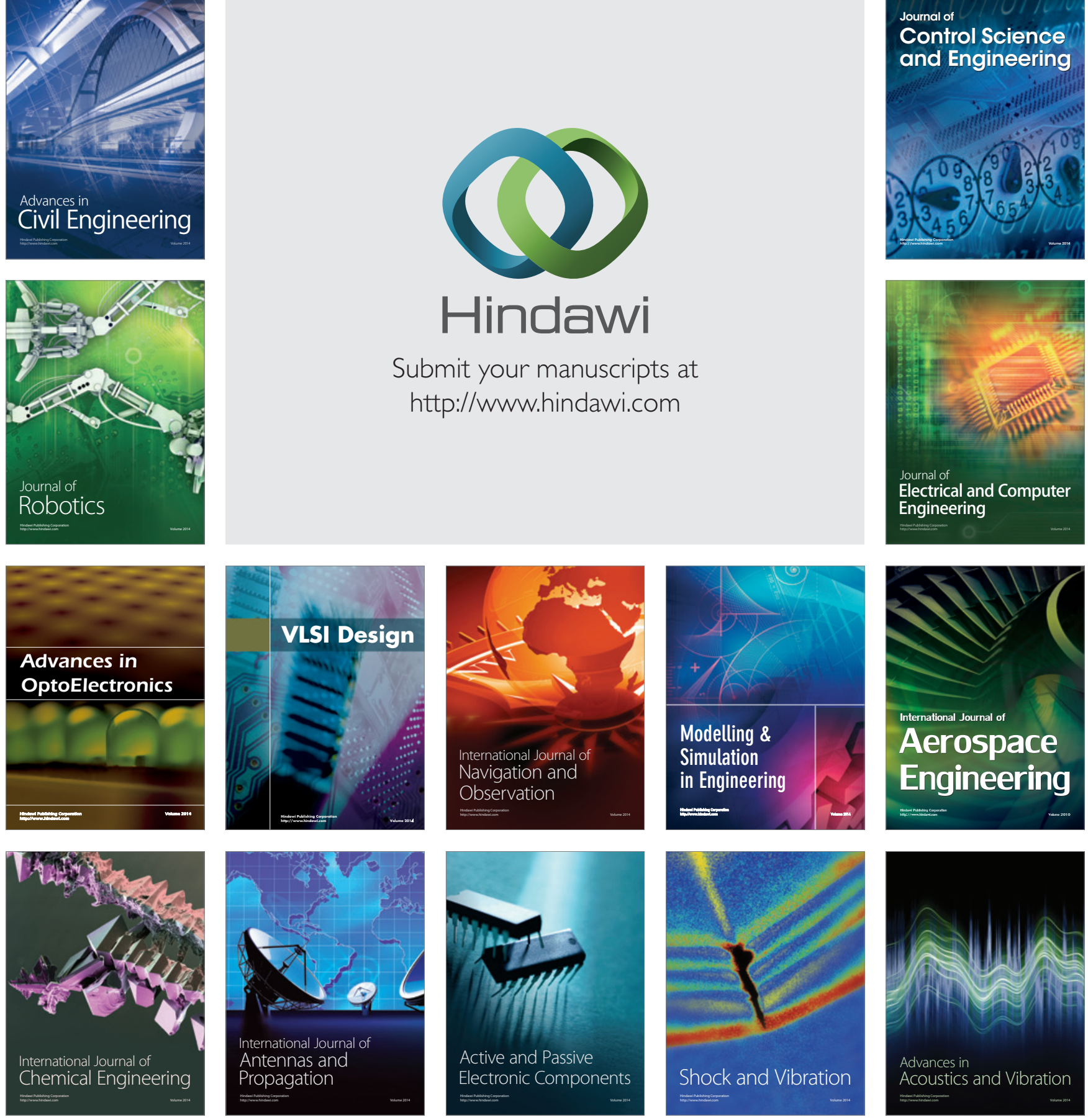